\title{
Image Denoising Using wavelet Transformation and Principal Component Analysis Using Local Pixel Grouping
}

\author{
D.Priyanka ${ }^{1}$ K.Praveena ${ }^{2}$ K.Praveena ${ }^{3}$ V.H.N Mounika ${ }^{4}$ V.Hindumathi ${ }^{5}$ \\ ${ }^{1-4}$ (Department Of Electronics And Communication Engineering, BVRIT Hyderabad College Of Engineering \\ For Women/JNTUH, India) \\ ${ }_{5}^{5}$ Assistant Professor In Department Of Electronics And Communication Engineering, BVRIT Hyderabad \\ College Of Engineering For Women/JNTUH, India)
}

\begin{abstract}
The image may be corrupted with noise during the process of image acquisition or transmission. Denosing is the process of removing the noise from the corrupted image. Denoising of the image ia an important step in the image restoration process. This paper describes two efficient denoising techniques, wavelet transformation and Principal Component analysis using Local Pixel Grouping. The wavelet transformation is the technique in which the input signal is divided into multiple scales or sub-bands which contains different time frequency components. At each scale, thresholding is performed to suppress noise. This technique $i$ shaving a disadvantage of edge distortion and PCA using LPG overcomes this advantage.

$P C A$ is a de-correlation technique in statistical signal processing which is mainly used in dimensionality reduction and pattern recognition. In Principal Component Analysis, a pixel and its nearest are grouped as vector variables called Local Pixel Grouping. During LPG, the trivial information is removed by compressing the data which helps in finding the patterns by preserving the edges of the image. PCA is a useful technique for finding the patterns in the data of high dimensions. The Peak Signal to Noise Ratio(PSNR) values of both the techniques are calculated.
\end{abstract}

Keywords: Local Pixel Grouping $(L P G)$, Peak Signal to noise ratio(PSNR), Principal Component Analysis(PCA), Thresholding, Wavelet transformation.

\section{Introduction}

During the process of image acquisition every image is prone to certain amount of noise. The image acquisition is done with the help of hardware and due to the inefficient functionality of the hardware some amount of noise gets added. Noise is added even during transmission where the unwanted data interferes with the original image data. Image must undergo processing before it is used in any application. There are many methods used for denoising of the image in order to get high quality image. In this paper, two effective techniques are used namely wavelet transformation and PCA using Local Pixel Grouping.

In wavelet technique, first the image undergoes decomposition and then the thresholding is performed in order to retain the significant image coefficients. For reconstruction of the original image, inverse wavelet transform is applied where the noise gets separated from the original image. The disadvantage with this wavelet technique is that the edges are not restored properly, so PCA using LPG is used to overcome this disadvantage. In PCA, the image data is compressed by using LPG for finding the patterns of the data and the datasets obtained after LPG are transformed into PCA domain where the noise along with the insignificant data is removed. Here, PCA is performed in two stages. In the first stage, most of the noise is removed and for further refining of the image second stage can be implemented. In this paper, from the experimental results obtained we have concluded by finding the efficiency of both the techniques by comparing the PSNR values and from the resultant outputs.

\section{Wavelet Transformation}

An image may be defined as a two-dimensional function $f(x, y)$, where $x$ and $y$ plane coordinates and the amplitude of $f$ at any pair of coordinates $(x, y)$ is called the intensity or gray level of the image at that point. Based on the Cartesian coordinate system, in the RGB model each color appears in its primary spectral components of red, green and blue [1]. Wavelet transformation is capable of providing time and frequency information simultaneously by giving the time-frequency representation of the signals. A wavelet is a wave like oscillation with an amplitude that begins at zero, increases and then decreases back to zero. As a mathematical tool, wavelets can be used to extract information from many different kinds of data and is not limited to audio signals and images [3]. Wavelet transform decomposes the image into several components based on the frequency characteristics of the signal. These components are converted into wavelet coefficients which are distributed into sub-bands depending upon the frequencies. These sub-bands are produced by passing the image 
through the filters. Then, the thresholding is performed on the obtained coefficient values to remove the insignificant values (noise).

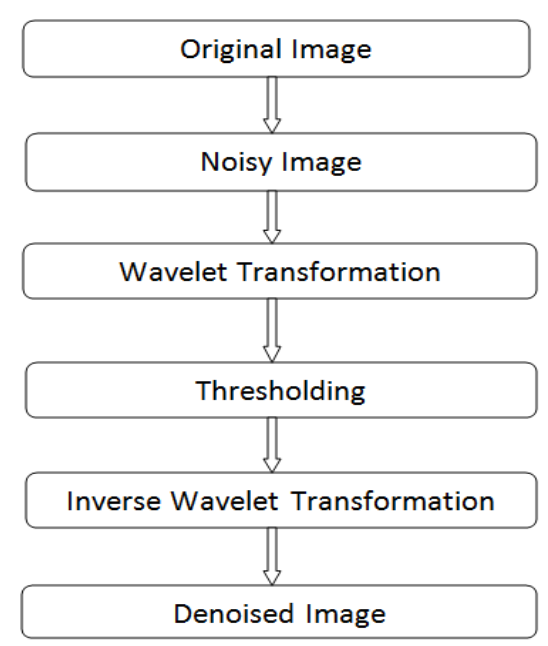

Fig.1: Wavelet Denoising Algorithm

In thresholding, the noisy coefficients are removed. Finally, inverse transformation is applied on the coefficients obtained after thresholding to reconstruct the original image i.e. de noised image without any loss of information.

Wavelet transformation for image denoising consists of three steps [2][4]

- In the first step, wavelet transformation is applied to the image.

- In the second step, wavelet coefficients undergo thresholding.

- In the third step, inverse wavelet transformation is applied for reconstructing the image.

In wavelet transformation, the image which is corrupted with noise when passed through two complementary filters gives approximation sub-signal and detail sub-signal [4]. Again the approximation and detail sub-signals are passed through the filters produces approximation(LL), vertical(LH), horizontal(HL) and diagonal $(\mathrm{HH})$ sub-bands.

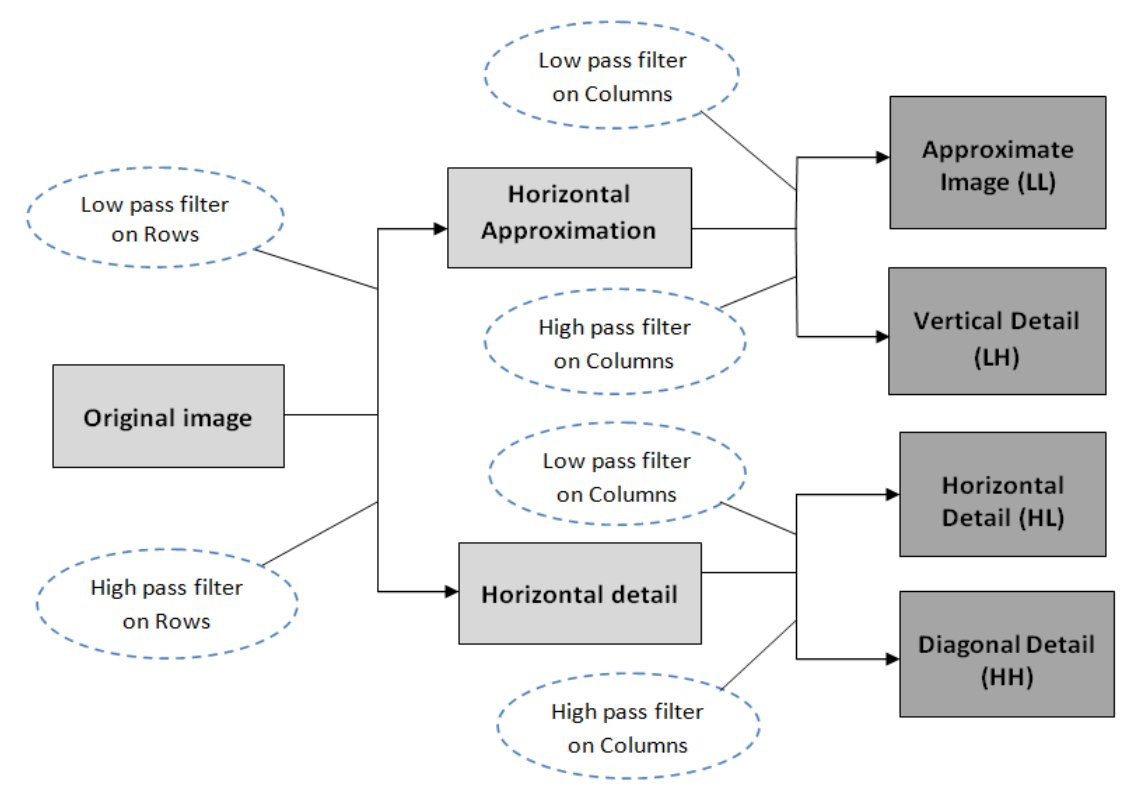

Fig.2: Block diagram of Wavelet Transformation

The sub-signal(approximation) produced from the low pass filter will have a highest frequency equal to half of the original signal. According to the Nyquist sampling this change in frequency range means that only half of original samples need to be kept in order to perfectly reconstruct the signal. If the details of vertical, horizontal and diagonal sub-bands are small, they can be set to zero without significant change in image. Hence 
filtering and compression can be achieved.

Thresholding is performed to eliminate the noisy components. It removes noise by killing coefficients that are insignificant insignificant relative to some threshold. There are two types of thresholding, hard and soft. Hard thresholding is a keep and kill procedure. If the signal is below threshold output is zero and it is above threshold then output is U (Input Signal). Soft thresholding shrinks coefficients above the threshold in absolute value [5].

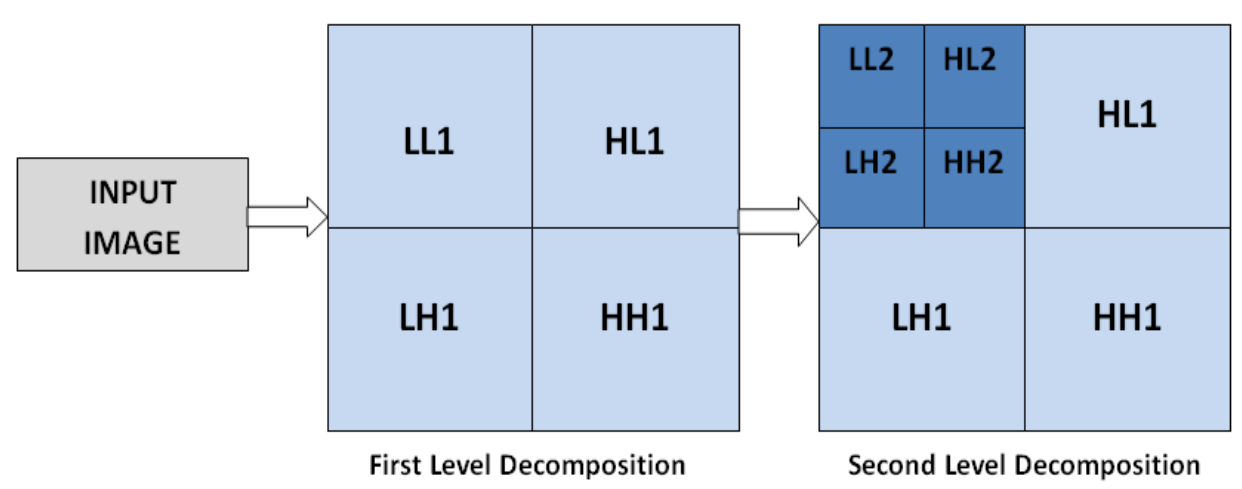

Fig.3: First and Second level of Image Decomposition

Thresholding plays an important role in the denoising process. A large threshold value leads to loss in required image coefficients and a small threshold value holds the noisy coefficients [4]. Inverse discrete wavelet transformation is applied for the reconstruction of the original image without loss of information.

\section{Pca Lpg Denoising Algorithm}

PCA is a statistical technique used to reduce the dimensionality of a dataset consisting of large number of interrelated variables while retaining as much as possible of the variation present in data set. This reduction is achieved by transforming to a new set of variable, the principal components which are uncorrelated and which are ordered so that the first few retain the most of the variation present in all of the original variables [13][14]. It is a de-correlation technique that extracts relevant information from confusing data.

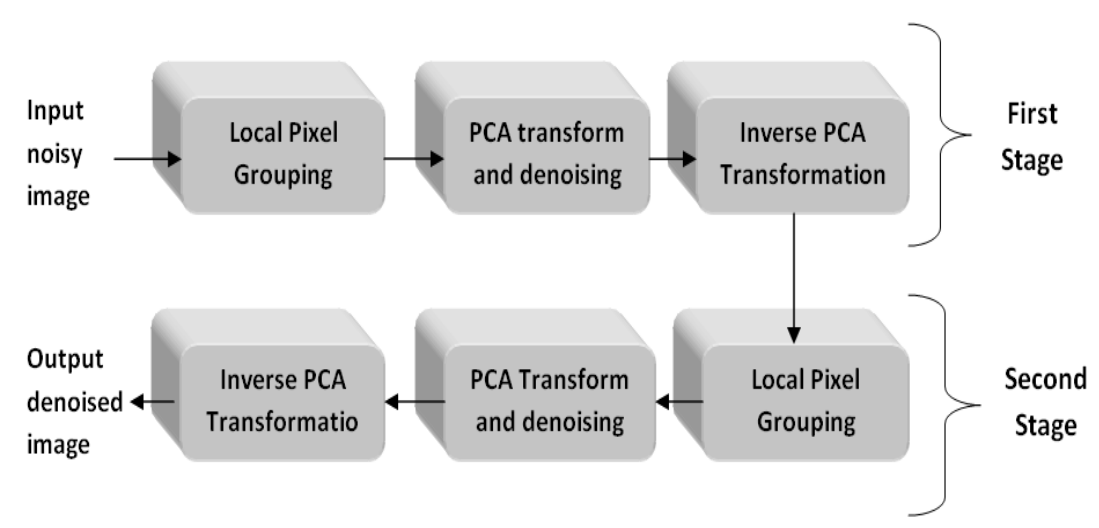

Fig.4: Block Diagram of LPG PCA Algorithm

This technique is mainly used in pattern recognition and data compression. Here we use PCA denoising method with LPG for noise removal [6]. In LPG-PCA scheme we make a vector variable by grouping the pixels with similar local spatial structures.

In Fig.4 shows the flowchart of two stage PCA using LPG. First, an image prone with noise is taken and Local Pixel grouping is performed. In the next step, It then transforms original dataset into linearly uncorrelated PCA domain and converse only the most significant principal aomponents where the noise and the trivial information can be removed. Finally, inverse PCA transformation is employed for image restoration.

PCA is a way of identifying patterns in data and expressing the data in such a way as to highlight its similarities and differences [15]. 
The algorithm consists of two stages, in the first stage the noise is significantly removed, the LPG accuracy will be much improved in the second stage by refining the output of the first image so that the second stage denoising result is visually much better [7].

\subsection{Principle Component Analysis}

The dataset is represented by the matrix $\mathbf{x}=\left[\begin{array}{llll}x_{1} & x_{2} & \ldots & \ldots\end{array} x_{m}^{T}\right.$ an m-component vector variable denoted by

$$
\mathbf{X}=\left[\begin{array}{ccccc}
x_{1}^{1} & x_{1}^{2} & x_{1}^{3} & \cdots & x_{1}^{n} \\
x_{2}^{1} & x_{2}^{2} & x_{2}^{3} & \cdots & x_{2}^{n} \\
x_{m}^{1} & x_{m}^{2} & x_{m}^{3} & \cdots & x_{m}^{n}
\end{array}\right]
$$

and the sample matrix $\mathbf{x}$, in $x_{i}^{j}, j=1,2,3, \ldots . n$ are the discrete samples of variable and $i=1,2,3 \ldots . n$. The $i$ th row of sample matrix $\mathbf{X}$, denoted by $X_{i}=\left[\begin{array}{lllll}x_{i}^{1} & x_{i}^{2} & x_{i}^{3} & \cdots & x_{i}^{n}\end{array}\right]$ is called the sample vector of $x_{i}$ * The mean value of $X_{i}$ is calculated as

$$
\mu_{i}=\frac{1}{n} \sum_{j=1}^{n} X_{i}(j)
$$

And then the sample vector $X_{i}$ is centralized as $\bar{X}_{i}=X_{i}-\mu_{i}=\left[\begin{array}{llll}\bar{x}_{i}^{1} & \bar{x}_{i}^{2} \bar{x}_{i}^{3} & \cdots & \bar{x}_{i}^{n}\end{array}\right]$ where $\bar{x}_{i}^{j}=x_{i}^{j}-\mu_{i}$.

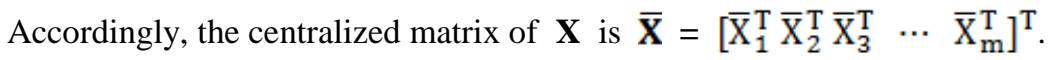

The covariance matrix is calculated as :

$$
\mathbf{\Omega}=\frac{1}{n}{\overline{X X^{T}}}^{T}
$$

The goal of PCA is to find an orthogonal transformation matrix $\mathbf{P}$ to de-correlate $\overline{\boldsymbol{X}}$, i.e. $\overline{\boldsymbol{Y}}=\boldsymbol{P} \overline{\boldsymbol{X}}$ so that the co-variance matrix of $\overline{\boldsymbol{Y}}$ is diagonal. Since the co-variance matrix $\Omega$ is symmetrical, it can be written as :

$$
\mathbf{\Omega}=\emptyset \boldsymbol{\Lambda} \emptyset^{\mathbf{T}}
$$

Where $\emptyset=\left[\emptyset_{1} \emptyset_{2} \cdots \emptyset_{m}\right]$ is the $m x m$ orthonormal eigen vector matrix and $\boldsymbol{\Lambda}=\operatorname{diag}\left\{\lambda_{1}, \lambda_{2}, \ldots, \lambda_{m}\right\}$ is the diagonal eigenvalue matrix with $\lambda_{1} \geq \lambda_{2} \geq \cdots \geq \lambda_{m}$. The terms $\emptyset_{1}, \emptyset_{2}, \ldots, \emptyset_{m}$ and $\lambda_{1}, \lambda_{2}, \ldots, \lambda_{m}$ are the eigenvectors and eigenvalues of $\Omega$. By setting $\mathbf{P}=\emptyset^{T}$.

$$
\overline{\boldsymbol{X}} \text { can be decorrelated, i.e. } \overline{\boldsymbol{Y}}=\boldsymbol{P} \overline{\boldsymbol{X}} \text { and } \boldsymbol{\Lambda}=\frac{\mathbf{1}}{\boldsymbol{Y}} \overline{\boldsymbol{Y}}^{T} \text {. }
$$

An important property of PCA is that it fully de-correlates the original dataset $\overline{\boldsymbol{X}}$. The energy of the signal concentrates on a small subset of the PCA transformed dataset and the energy of the noise will evenly spread over the whole dataset. So, the signal and noise can be better distinguished in the PCA domain [8].

\subsection{Local Pixel Grouping}

Local pixel grouping is the algorithm to remove noise from images using principal component analysis. It is based on the assumption that the energy of a signal will concentrate on a small subset of the PCA transformed dataset, while the energy of noise will evenly spread over the whole dataset.

The original image is denoted by $\boldsymbol{I}$ and noise is denoted by $\vartheta$, the measured image will be $\boldsymbol{I}_{\vartheta}=\boldsymbol{I}+\vartheta$. In order to denoising $\boldsymbol{I}_{\vartheta}$, a trained dataset $\boldsymbol{X}_{\vartheta}$ is constructed and used and by applying PCA the noise of the image can be reduced [9].

A pixel and its neighbors are modeled as a vector variable as shown in the Fig:5. The training samples of this variable are selected by identifying the pixels with self similarity based local spatial structures to the underlying one in the local window. The local structural statistics of the variables can be accurately computed so that the image edge structures can be well preserved after shrinkage in the PCA domain for noise removal.

For each pixel in the image, select a $K X K$ window and the training window is $L X L$, where $L>K$. Take the pixels in each possible $K X K$ block within the $L X L$ training block yields $(L-K+1)^{2}$ samples. LPG makes more difference between noise and signal from the locally grouped image blocks. LPG-PCA method is spatially adaptive image representation so that it can better characterize the image local structures. 


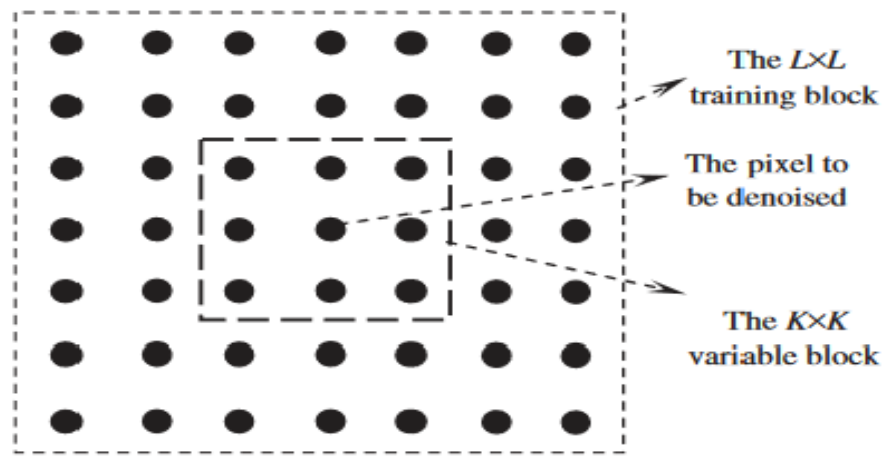

Fig.5: Representation of surrounding pixel values

There are two reasons for noise residual. First, because of the strong noise in the original dataset, the covariance matrix is much corrupted with noise which leads to the estimation bias of the PCA transformation matrix and hence deteriorates the denoising performance. Second, the strong noise in the original dataset will also lead to LPG errors, which leads to the estimation bias of the covariance matrix. So, it is necessary for further denoising of the output.

Since the noise has been much removed in the first round of LPG-PCA denoising, the LPG accuracy and estimation of covariance matrix can be much improved with the denoised image. Thus we can implement the LPG-PCA denoising procedure for the second round to enhance the denoising results.

\section{Comparison Between Wavelet And Pca Lpg}

Wavelet Transform though effective in noise removal is non adaptive hence does not yield desired results on the real time image. The main disadvantage with wavelet transform is edges are not restored properly and causes distortion in the denoised image. To overcome the drawback, principal component analysis is used.

\begin{tabular}{|c|c|c|c|}
\hline \multirow{2}{*}{ S.No } & Wavelet Transformation & \multicolumn{2}{|c|}{ PCA LPG } \\
\cline { 3 - 4 } & & Level 1 & Level 2 \\
\hline 1. & 28.3 & 32 & 33 \\
\hline 2. & 26 & 29.6 & 30 \\
\hline 3. & 27.5 & 29.4 & 29.7 \\
\hline
\end{tabular}

Table 1: Comparison of PSNR values for Wavelet and PCA LPG PSNR (Peak Signal to Noise Ratio)

It is the ratio between maximum possible power of the signal to the power of corrupting noise that affects fidelity of representation.

$$
\text { PSNR }=10 \log _{10}\left(\frac{m_{\text {MSE }}^{2}}{\text { MSE }}\right)
$$

Where, $\max ^{2}$ is the maximum power and MSE is mean square error

\section{Results}

For Image-1

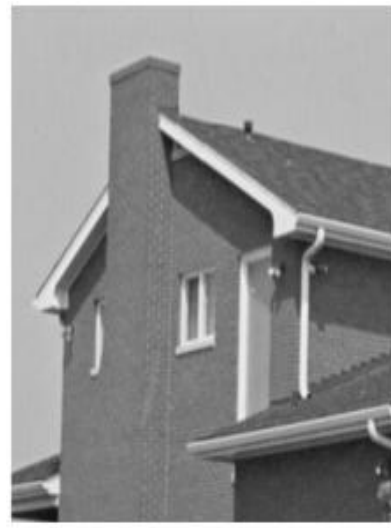

(Original Image)

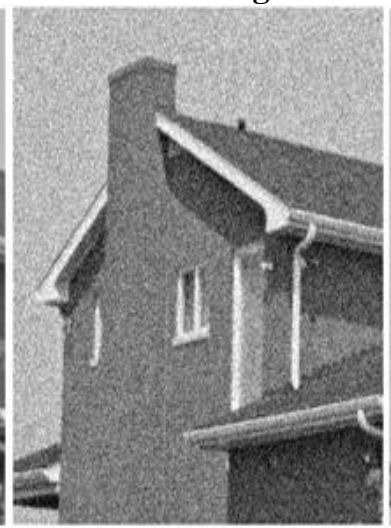

(Noisy Image)

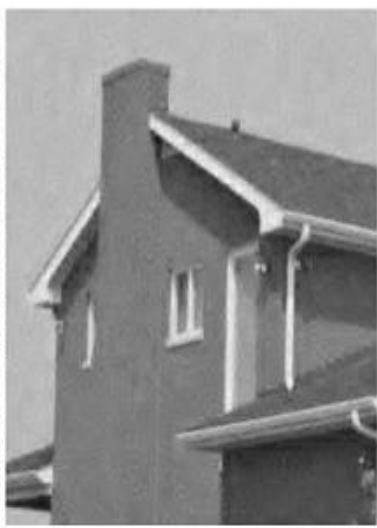

(Wavelet Output)

Fig.6: Image1- Results of Wavelet Transformation 


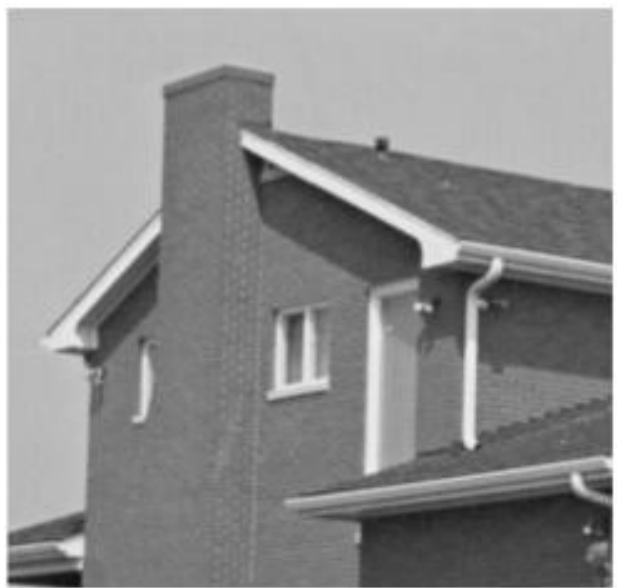

(Original Image)

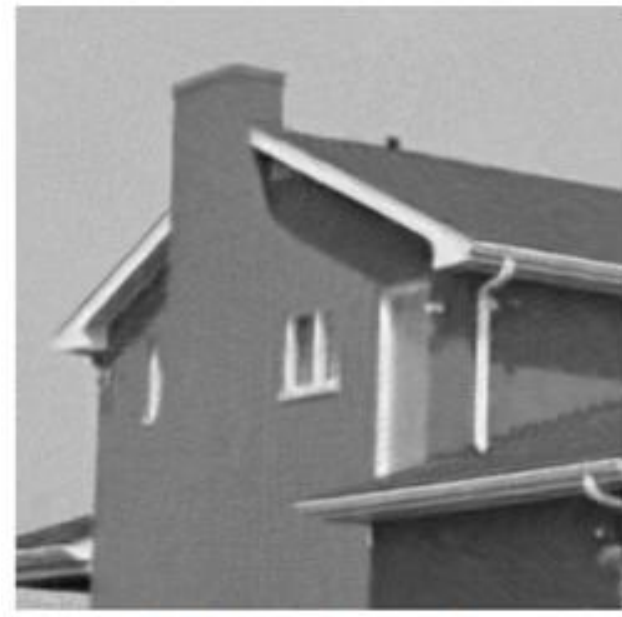

(First stage output)

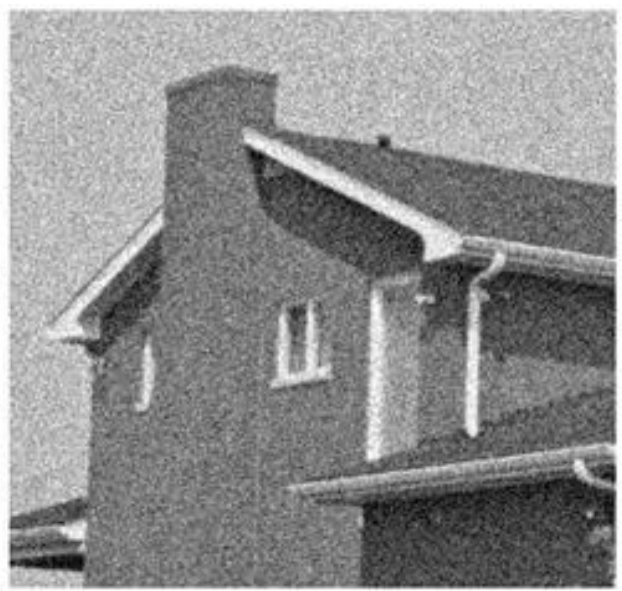

(Noisy Image)

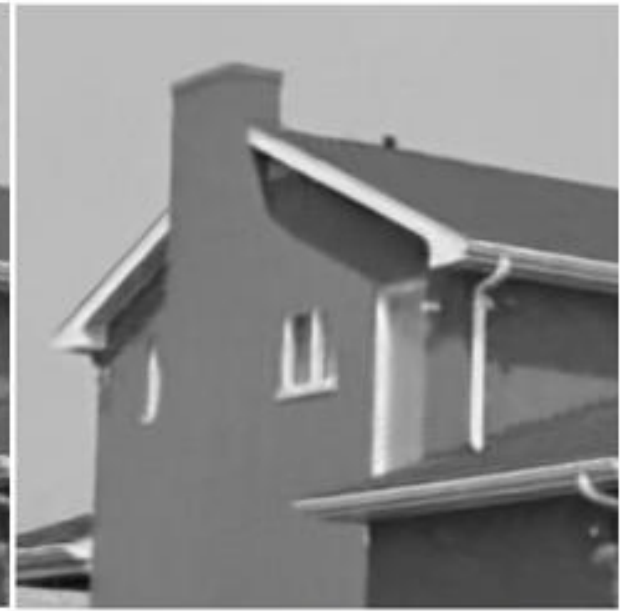

(Second stage output)

Fig.7: Image1- Results of first stage and second stage PCA using LPG For Image-2

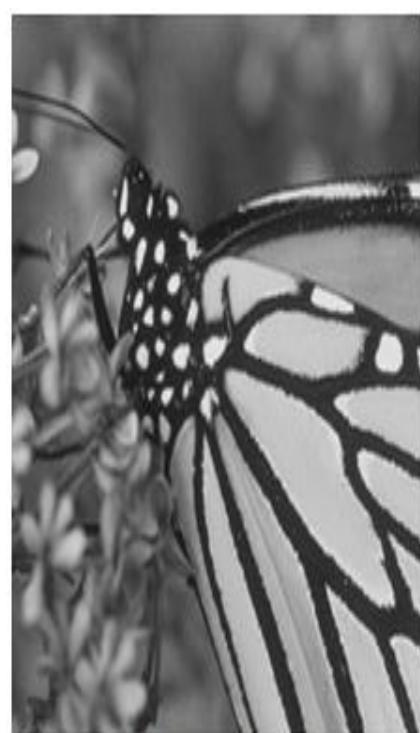

(Original Image)

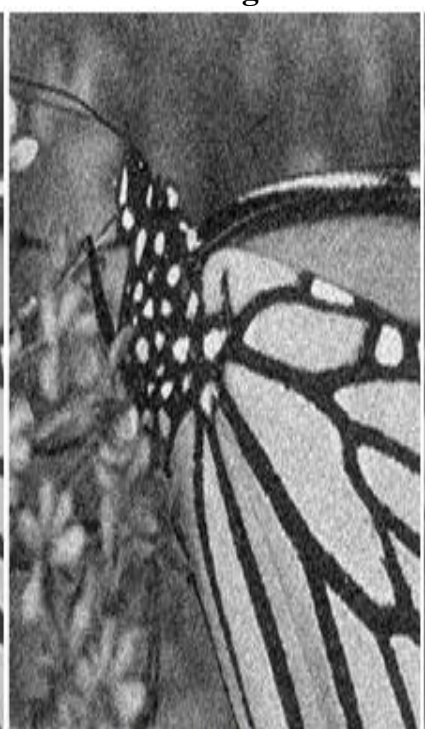

(Noisy Image)

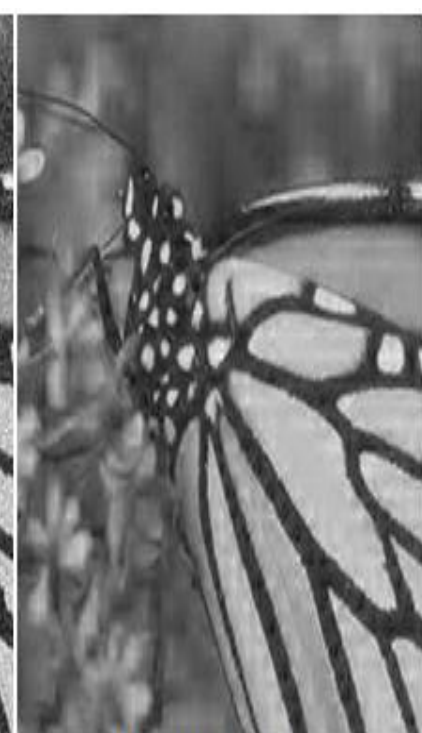

(WaveletOutput)

Fig.8: Image2- Results of Wavelet Transformation 


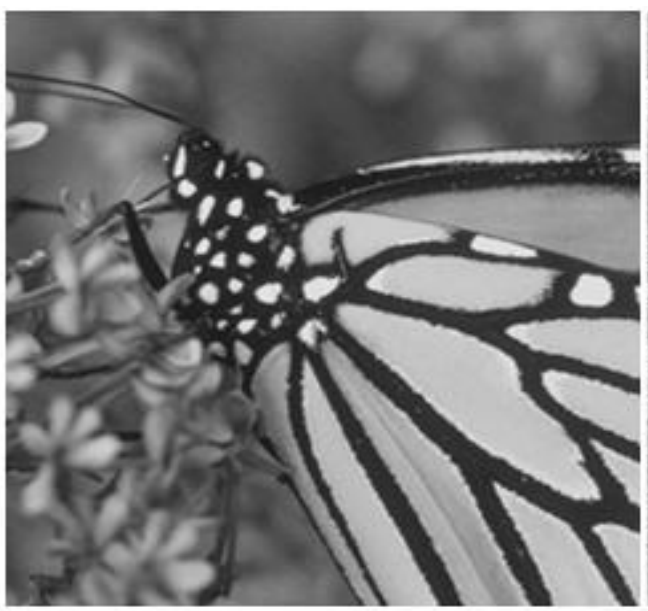

(Original Image)

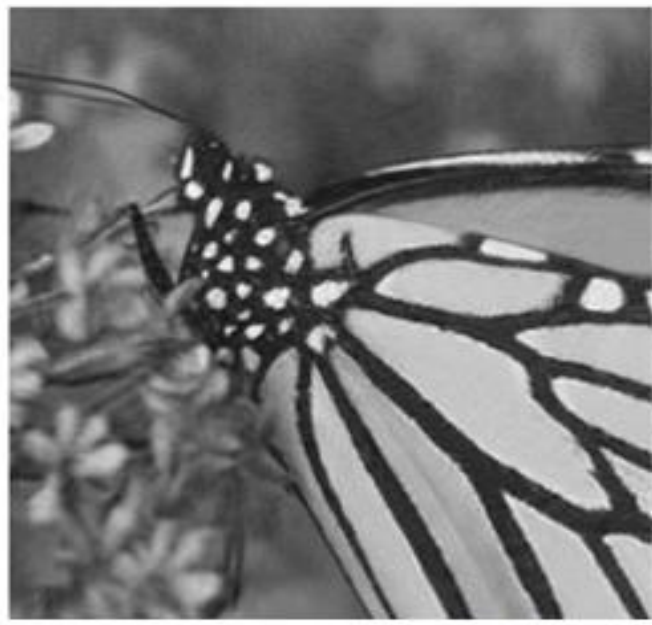

(First stage output)

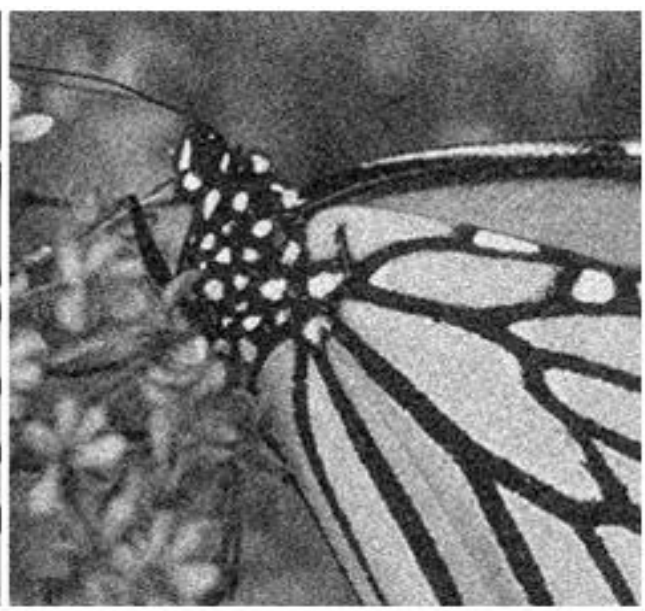

(Noisy Image)

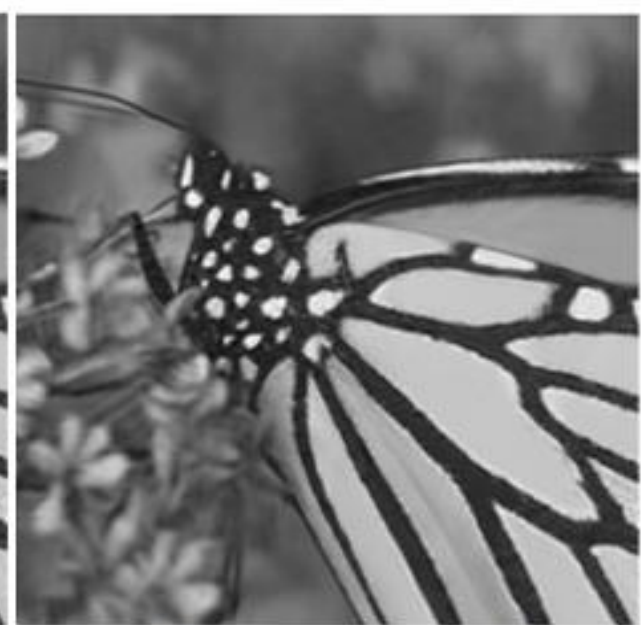

(Second stage output)

Fig.9: Image2- Results of first stage and second stage PCA using LPG

\section{Conclusion}

This paper deals with the techniques principal component analysis using local pixel grouping and wavelet transformation for image denoising. The de noised images obtained from both the techniques are compared. In PCA-LPG, to preserve the local image structures the pixel and its nearest neighbours are grouped as a vector variable and a block matching technique is used for transforming the required pixels into PCA domain. This de-noising technique is efficient in preserving features of an image when compared Wavelet Transformation.

\section{References}

[1]. R. C .Gonzalez, R.E. Woods, Digital Image Processing, second edition., Prentice- Hall,EnglewoodCliffs, NJ, 2002.

[2]. Sameer Khedkar, Kalyani Akant and Milind M. Khanapurkar, "Image denoising using wavelet transform" - IJRET Journal, vol-5, 2016.

[3]. Wavelet - Wikipedia, the free encyclopedia available online at : https://en.wikipedia.org/wiki/Wavelet.

[4]. S.Kother Mohideen, Dr.S.Arumuga Perumal and Dr.M.Mohamed Sathik, "Image De-noising using discrete wavelet transform " - IJCSNS International Journal, vol-8, 2008.

[5]. P.C.V. Amulya and Sai Vijaya Laxmi, "Image denoising with wavelet based thresholding "- IOSR Journal, vol-18, pp 01-04, 2016.

[6]. Y.Murali Mohan Babu, Dr. M.V. Subramanyam, Dr. M.N. Giri Prasad, "PCA based image denoising" - SIPIJ International journal, vol-3, 2012.

[7]. Sangeetha Rani and Pankaj Dev Chadha, "Principal component based image de-noising using local pixel grouping", IJSRD, vol-4, 2016.

[8]. Lei Zhang, Weisheng Dong, David Zhang, Guangming Shi, "Two-stage image denoising by principal component analysis with local pixel grouping" Elsevier-Pattern recognition, vol-43, 1531-1549, 2010. 
Image Denoising Using Wavelet Transformation and Principal Component Analysis .....

[9]. Pixel Grouping - Wikipedia, the free encyclopedia available online at : https://en.wikipedia.org/wiki/Local_pixel_grouping.

[10]. Sabita Pal, Rina Mahakud, Madhusmita Sahoo,"PCA based Image denosing using LPG", IJCA special issue on $2^{\text {nd }}$ national conference- CCSN, 2011.

[11]. K. John Peter, Dr. K. Senthamari Kannan, Dr. S. Arumugan and G. Nagarajan, "Two-stage denoising by Principal Component Analysis with self similarity pixel Strategy ”, IJCSNS, vol-11 No.5, 2011.

[12]. Rachana A. Dhannawat and Archana B. Patankar, "Image denoising by using Local Pixel Grouping with PCA and SVD along with JBF and Median filters", (IJAIS) - ISSN : 2249-0868, 2016.

[13]. Mr.Ankur N.Shah,'Introduction to Wavelet transform and two stage image denoising using Principal Component Analysis with Local Pixel Grouping (LPGPCA) method ”, IJSRD, vol-1 Issue 4, 2013.

[14]. I.T. Jolliffe, "Principal Component Analysis", second edition, Springer.

[15]. Divesh Kumar and Dheeraj Kalra, "principal Component Analysis image denoising using Local Pixel Grouping", ICRISTME, 2016. 